\title{
Seroconversion and adverse events in the first month after the administration of two doses of the inactivated SARS-CoV-2 vaccine in Turkey: Is a booster dose required?
}

\author{
Burak Mete ${ }^{1}$, Ferdi Tanir ${ }^{1}$, Hakan Demirhindi ${ }^{1}$, Ertan Kara ${ }^{1}$, Filiz Kibar ${ }^{1}$, Salih Cetiner $^{1}$, \\ Aslihan Candevir-Ulu ${ }^{1}$, and Rabia Dal ${ }^{1}$ \\ ${ }^{1}$ Cukurova University Faculty of Medicine
}

June 7, 2021

\begin{abstract}
Aims: Limited information is available about the efficacy and adverse events of COVID-19 vaccines introduced into public use with Emergency Usage Licences without completing phase-3 trials. Data refer to healthy and mostly younger people, with lacking evidence about the protectivity. This study aimed determining seroconversion rates and levels specific to anti-S-RBD IgG and total anti-spike/anti-nucleocapsid IgM and IgG antibodies against SARS-CoV-2 after the administration of two doses of the inactivated SARS-CoV-2 vaccine in Turkey, comparing three types of antibodies. It was also aimed to assess short-term adverse events due to the vaccine. It is intended to answer the questions about efficacy and safety, which lack in phase trials, especially at community level. It is also aimed to collect data, which will form the basis for assessing whether antibodies are protective at different community settings. Methods: The study carried out in Turkey, comprised 282 healthcare workers who received two doses of the inactivated SARS-CoV-2 vaccine administered in two $3 \mu \mathrm{g}$ doses, 28 days apart. In day-28 after the second dose, anti-S-RBD IgG and total anti-spike/anti-nucleocapsid IgM and IgG antibodies against SARS-CoV-2 were detected by in-vitro chemiluminescence immunoassay. Results: At day 28 after the second dose, the seroconversion rates were found to be $92.9 \%$ for total anti-spike/anti-nucleocapsid IgG and $15.2 \%$ for IgM and $98.2 \%$ for anti-S-RBD IgG antibodies. The immunogenicity was affected by pre-vaccination natural COVID-19 history and age. The incidence of at least one adverse event was found as $29.8 \%$ after the first dose and $24.1 \%$ after the second dose, with the most common events being pain at the injection site, weakness, fatigue and headache. Conclusion: A high rate of seroconversion was observed with no serious adverse events. Prior natural COVID-19 history and age significantly contributed to strong immunogenicity. A booster dose appears required for older ages and individuals without immune response.
\end{abstract}

\section{Hosted file}

IJCM---Main Text-Seroconversion after inactivated SARS-CoV-2 vaccine.docx available at https: //authorea.com/users/415437/articles/525209-seroconversion-and-adverse-events-inthe-first-month-after-the-administration-of-two-doses-of-the-inactivated-sars-cov-2vaccine-in-turkey-is-a-booster-dose-required 


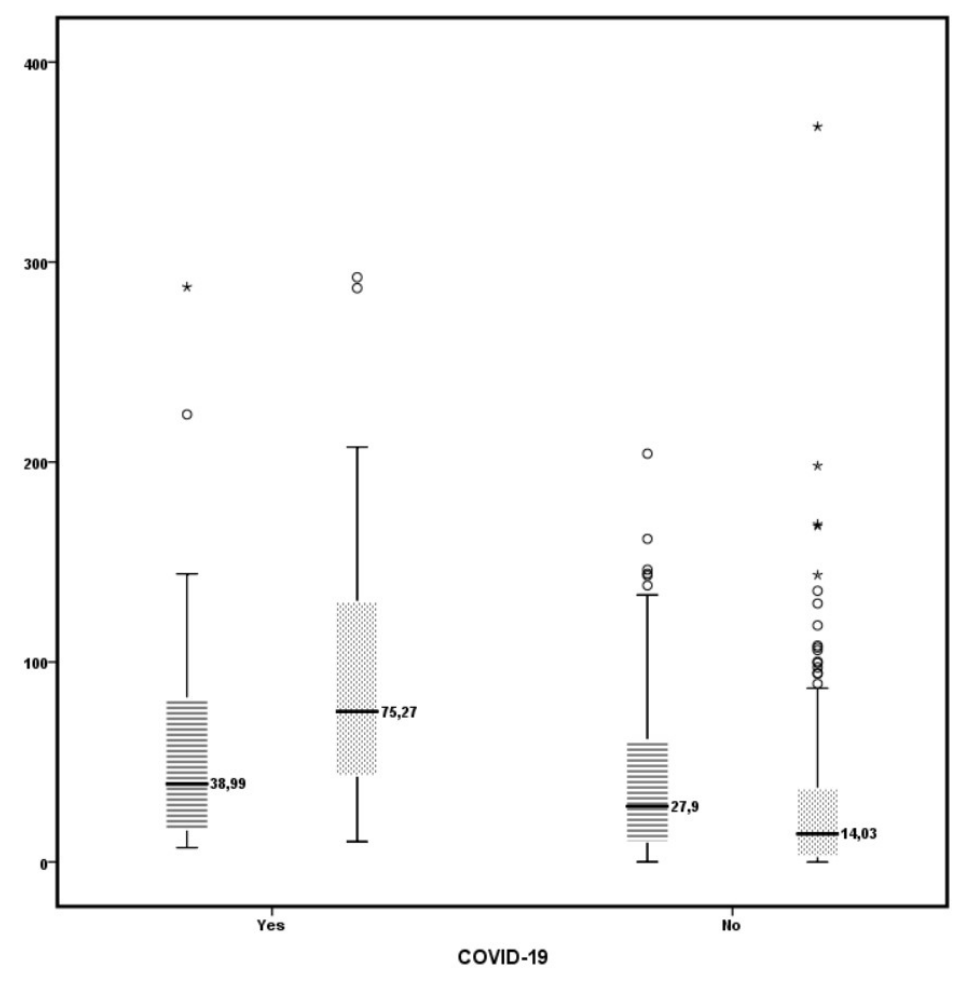

$=$ Anti-S RBD IgG

Anti-Spike and Anti-
Nucleocapsid lgG

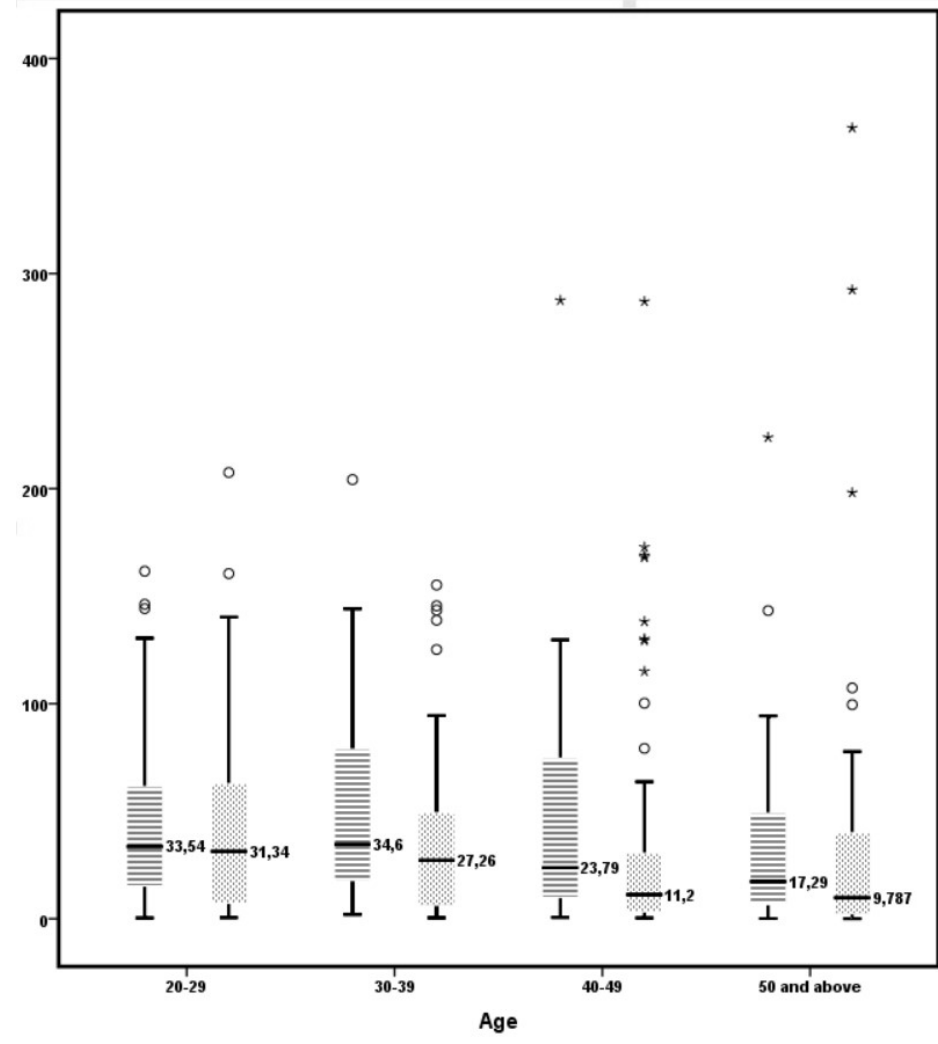

$=$ Anti-S RBD IgG Anti-Spike and Ant-
Mucleocapsid IgG 